\title{
Evandro Chagas e seus hospitais: dirimindo a polêmica acerca do Hospital do Instituto Evandro Chagas em Belém, Estado do Pará, Brasil
}

\section{Evandro Chagas and his hospitals: settling the controversy over the Hospital do Instituto Evandro Chagas in Belém, Pará State, Brazil}

\author{
Manoel do Carmo Pereira Soares, Heloisa Marceliano Nunes
}

Instituto Evandro Chagas/SVS/MS, Seção de Hepatologia, Belém, Pará, Brasil

\section{RESUMO}

Este artigo, em meio à importância contextual dos hospitais, tem por objetivo contribuir para o esclarecimento de questões referentes à criação, atuação e desativação do Hospital do Instituto Evandro Chagas em Belém, Estado do Pará, Brasil, durante os anos 40 do século XX. Trata, portanto, de uma situação que remete a reflexões vinculadas à memória do Instituto Evandro Chagas e às perspectivas para a atuação institucional integral, desde os tempos de Instituto de Pathologia Experimental do Norte.

Palavras-chave: História da Medicina; História da Saúde Pública; Medicina Tropical.

\begin{abstract}
Based on the importance of hospitals, this article aims to examine the foundation, functioning, and closing of the Hospital do Instituto Evandro Chagas, located in Belém, Pará State, Brazil, in the 1940s. The article reflects on a particular aspect of the history of the Instituto Evandro Chagas and how it has led to the progress of integrated institutional work since the times of the Instituto de Pathologia Experimental do Norte.
\end{abstract}

Keywords: History of Medicine; History of Public Health; Tropical Medicine.

\section{INTRODUÇÃO}

Não se pode falar ou atuar na saúde sem alguma referência aos hospitais. Em nível nacional, e mesmo nas Américas, consta que o primeiro hospital construído foi a Santa Casa de Misericórdia de Todos os Santos, por iniciativa do português Braz Cubas, em 1543, no antigo povoado de Enguaguaçu, Estado de São Paulo'. É essa, aliás, uma das possibilidades levantadas para que aquele povoado viesse a ganhar o nome atual Cidade de Santos. Constata-se, assim, como a história dos hospitais emerge de uma pactuação com o religioso. Cabe ainda registrar que, no Brasil daquela época, os jesuítas exerciam ocasionalmente atividades de médicos, enfermeiros, farmacêuticos, etc. Apenas posteriormente chegariam os profissionais da saúde.

Decerto, muito também se pode falar e discutir acerca dos hospitais, nos dias que atravessamos, e sobre as perspectivas afins; o que ganhamos e o que perdemos. Mas - e para nossos propósitos imediatos - o que Evandro (Serafim Lobo) Chagas tem a ver com isso? Bastante. Bastante mesmo. Foi um dos mais dedicados internos do Hospital de Manguinhos, no Estado do Rio de Janeiro, desde quando esse ainda ostentava o nome de Oswaldo Cruz, recebido em 1917, após a morte de Oswaldo. Já como graduado, Evandro viria a ser destacado diretor desse Hospital, o que justificou, em 1942, a sua reinauguração com - nome de Hospital Evandro Chagas. Atualmente o Hospital alicerça um novo Instituto dentro da Fundação Oswaldo Cruz (Fiocruz) - o Instituto Nacional de Infectologia Evandro Chagas².

Ainda no âmbito da cadeira de Doenças Tropicais da antiga Faculdade de Medicina do Rio de Janeiro, Evandro participou das atividades médicas e didáticas, desde o internato, junto com o pai, Carlos Chagas, e demais colaboradores, no histórico Hospital São Francisco de Assis $^{3}$. Registre-se que, em 1943, em 
Natal, Estado do Rio Grande do Norte, foi inaugurado um bem aparelhado hospital público, que reverenciava o nome de Evandro Chagas, fruto de suas passagens e atuações pelo nordeste brasileiro.

\section{O HOSPITAL DO INSTITUTO EVANDRO CHAGAS DE BELÉM}

Evandro Chagas, como forte traço de herança do pai, era obstinado pela unidade entre o trabalho no campo, no ambulatório, no laboratório e no hospital. Atuou como gestor em todas essas frentes. Não é, pois, de se estranhar que, em 1936, ao desenhar o Instituto de Pathologia Experimental do Norte (Ipen), nome precursor do Instituto Evandro Chagas (IEC), ele definisse um hospital compondo a sua estrutura ${ }^{4}$. Desde a criação do Ipen, Evandro trabalhou com essa convicção. Morreu cedo, em 1940, sabemos, sem concretizar o sonho. Mas, três anos depois e por absoluta justiça, a 10 de novembro de 1943, exatamente no dia do sétimo aniversário do Instituto, coroando um convênio internacional, o Serviço Especial de Saúde Pública (SESP), em grande comemoração - contando com participação de autoridades locais, nacionais e internacionais - inaugurava o Hospital do Instituto Evandro Chagas.

resgate da documentação fotográfica do momento da inauguração, mostrada neste artigo, contribui para esclarecer antigas discussões sobre o nível de vinculação entre o IEC e esse Hospital (Figura 1).

Brito Bastos, em seu livro sobre a evolução histórica do SESP-FSESP5, faz considerações sobre o processo de aquisição e construção do aludido Hospital; em certo ponto diz: "Em 10 de novembro de 1943, o Hospital foi oficialmente inaugurado com o nome de Hospital Evandro Chagas [...]" $]^{115}$. Mais adiante, entretanto, escreve:

Um fato merece aqui um esclarecimento. Em diversos documentos antigos da própria Diretoria do Programa da Amazônia e em alguns Boletins do SESP, encontra-se - Hospital com o nome de Hospital de Doenças Tropicais. Outros documentos se referem a Hospital Evandro Chagas e finalmente outros, a Hospital de Belém. $\bigcirc$ projeto de criação, refere-se a "Operação e Manutenção do Hospital Evandro Chagas de Belém"(3), enquanto o projeto de fechamento de suas atividades se refere ao Hospital de Belém. (10) O Dr. Otávio Maroja, pesquisador do Instituto Evandro Chagas e, como dissemos anteriormente um dos primeiros servidores do Hospital, confirma que o Hospital foi criado com o nome de Hospital Evandro Chagas, mudando depois para Hospital de Belém.

E, logo na sequência, complementa Brito Bastos ${ }^{5}$ :

Um outro ponto controvertido, é quanto a posição administrativa do Hospital. O Boletim do SESP $n^{\circ} 01$ de janeiro de 1943, notificando a inauguração do Hospital se refere ao hospital do Instituto Evandro Chagas e no Boletim do SESP $\mathrm{n}^{\circ} 03$, de janeiro de 1944, vamos encontrar a mesma designação [...] Por outro lado, em uma publicação de autoria do Dr. Miguel Azevedo, ex-pesquisador do Instituto Evandro Chagas, é dito que o "Serviço Especial de Saúde Pública dotou o Instituto Evandro Chagas de um Hospital bem aparelhado" [...].

Leônidas Deane, ao escrever o histórico do Instituto Evandro Chagas, relata que "em 1943 o SESP dotou o Instituto de um pequeno, mas moderno Hospital [...] hospital esse que prestou grande serviço no treinamento dos médicos e auxiliares do SESP"6.

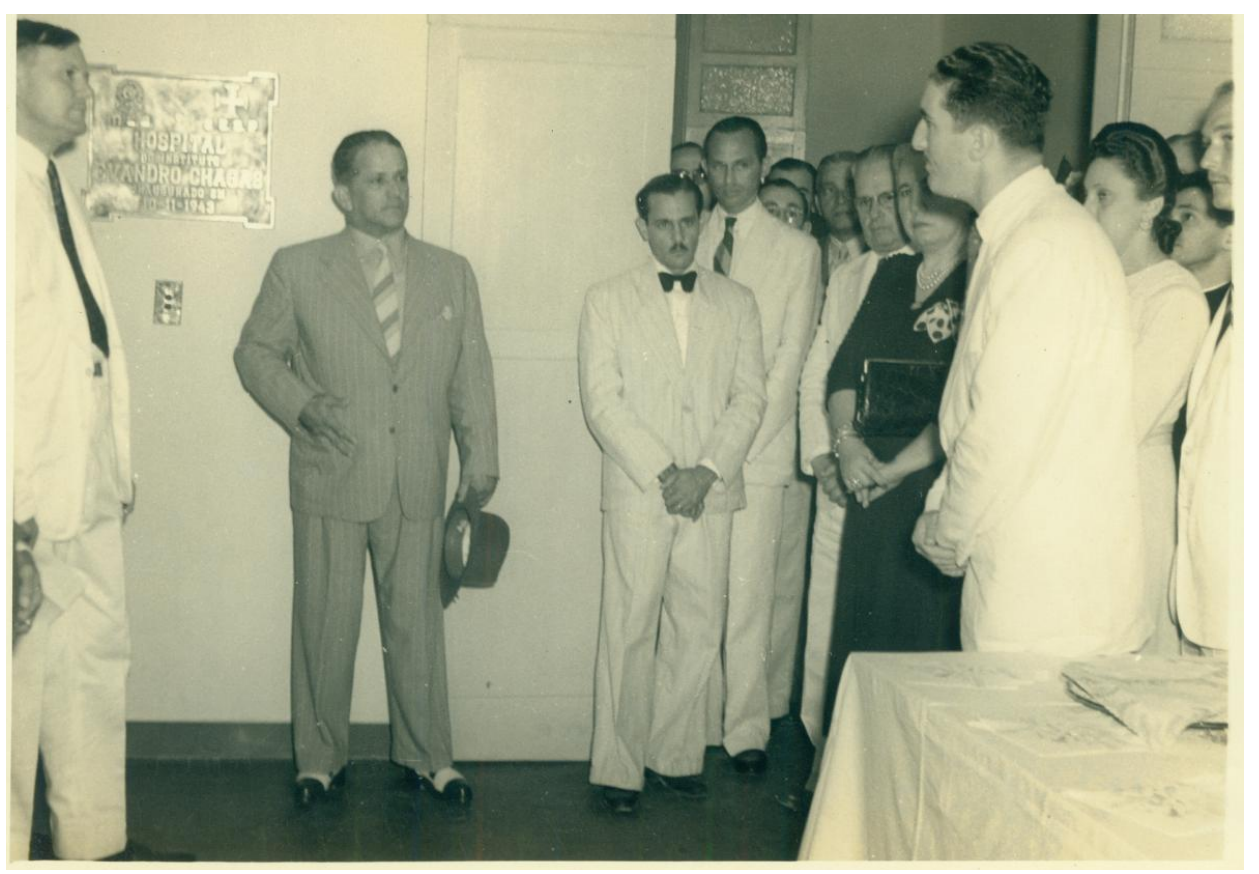

Fonte: Documento do acervo da Casa de Oswaldo Cruz/Fiocruz.

Figura 1 - Coronel Magalhães Barata, interventor federal no Estado do Pará, após descerrar a placa de inauguração do Hospital do Instituto Evandro Chagas, Cidade de Belém, Estado do Pará, a 10 de novembro de 1943 
Novamente recorremos a Brito Bastos ${ }^{5}$, que arremata:

Todas essas informações deixam clara a subordinação do Hospital de Belém ao Instituto Evandro Chagas. Mas, em nenhum dos projetos que tivemos oportunidade de consultar sobre o Hospital e sobre o Instituto, encontramos referência a tal subordinação administrativa. Não satisfeito, procuramos ouvir antigos médicos que trabalharam na Amazônia, como ○ Dr. Antonio Jorge de Almeida, Dr. Osvaldo José da Silva, ex-diretor do Programa da Amazônia, Dr. Isnard Teixeira, ex-diretor do Instituto Evandro Chagas, Dr. Orlando José da Silva, ex-diretor da Divisão de Educação Sanitária, e todos foram unânimes em negar tal subordinação. $O$ Dr. João Alves Meira, ex-diretor do Hospital, em carta que nos dirigiu, destaca a colaboração que sempre recebeu dos técnicos do IEC durante sua administração e diz textualmente "Fora esta colaboração destes cientistas, não havia outra ligação do Instituto Evandro Chagas e o Hospital: não havia qualquer subordinação do Hospital ao Instituto".

Um final triste para essa história é assim descrito ${ }^{5}$ :

Em 31 de dezembro de 1948, expirado o Acordo do $\mathrm{DNI}^{*}$ e a CCAW', o Hospital reverteu ao SESP e a 27 de janeiro de 1949 foi dado início ao seu fechamento sendo o prédio devolvido com todas as melhorias e grande parte dos equipamentos para serem usados no Hospital de Pronto Socorro da Cidade de Belém. Os programas de treinamento foram transferidos para o Hospital de Santarém e o restante do material distribuído com os Postos de Higiene ou Hospitais do SESP.

Não obstante o exposto, é essencial valorizar aquilo que se impõe como sentido maior, para além das letras e dos números exigidos pelo instante normativo e burocrático. A proposta do Instituto pedia um hospital especial e, em seus encaminhamentos afins, o SESP, que tantos hospitais já administrava, claramente dava sinais de anuência a tal propósito.
No mais, alguns registros parecem-nos suficientes para legitimar o passado do Hospital do IEC em Belém do Pará. Primeiro, o ritual de inauguração, na fotografia aqui mostrada, vê-se a presença do coronel Magalhães Barata, interventor federal no Estado do Pará, em frente ao grupo representativo da comunidade médica e científica, após descerrar a placa que ostentava as logomarcas do Ministério da Educação e Saúde (MES) e do SESP e que trazia escrito: "Hospital do Instituto Evandro Chagas inaugurado em 10-11-1943" (Figura 1). Isso somado à fotografia do Hospital em construção ${ }^{7}$ (Figura 2) e à elucidativa reportagem publicada no jornal carioca Correio da Manhã $^{8}$ (Figura 3), esse que, além de citar em longa e parcimoniosa reportagem a "inauguração do Hospital do 'Instituto Evandro Chagas"', diz em certo trecho que:

\begin{abstract}
Hospital que acaba de ser inaugurado é o complemento do antigo "Instituto de Patologia Experimental do Norte", hoje "Instituto Evandro Chagas". Quando esse jovem cientista morreu, deixou por ser realizado esse sonho: a instalação de um hospital de pesquizas em doenças tropicais. O SESP o realiza agora, e o novo hospital é, no gênero, o primeiro a ser construído em zona tropical no Brasil.
\end{abstract}

Relatórios dos anos subsequentes atestam o quanto ali se trabalhou, cuidou e produziu conhecimentos. A morte prematura de Evandro Chagas, bem como a construção fora do espaço físico das dependências do campus institucional, em terreno que pertencia à Santa Casa de Misericórdia do Pará, muito provavelmente contribuiu para o rompimento - talvez definitivo - do IEC com o seu Hospital. Os descaminhos éticos e administrativos, bem descritos por Brito Bastos ${ }^{5}$, que levaram à desativação desse empreendimento, levam-nos a reflexões de que devemos trilhar novos caminhos, porém priorizando, preservando e consolidando os passos dados, para não tropeçarmos. Sim, o IEC já teve um Hospital - próprio de pesquisa em doenças tropicais.

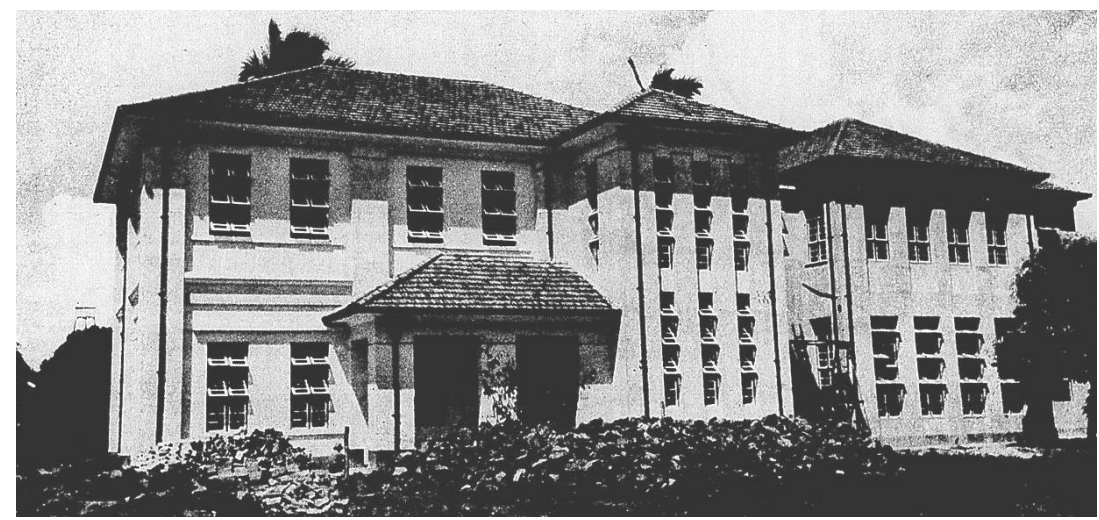

Fonte: $\bigcirc$ Observador Econômico e Financeiro. 1943 set;8(92):488.

Figura 2 - Aspecto do Hospital do Instituto Evandro Chagas, em período próximo à sua configuração final. Consta que esse Hospital ocupava uma área onde hoje se situa o Pronto Socorro Municipal, na atual Travessa 14 de março, Cidade de Belém, Estado do Pará

\footnotetext{
*DNI - Departamento Nacional de Imigração, instância do governo de Getúlio Vargas, financiado por um fundo especial da Rubber Development Corporation (RDC), em consonância com a CCAW e que se ocupava do recrutamento, encaminhamento, colocação e assistência de trabalhadores e familiares nos seringais da Amazônia brasileira? 
CORIREI0 I) I MANIIX - Quarta-feira, 1 de Dezembro de 19.43

\begin{tabular}{|c|c|c|}
\hline 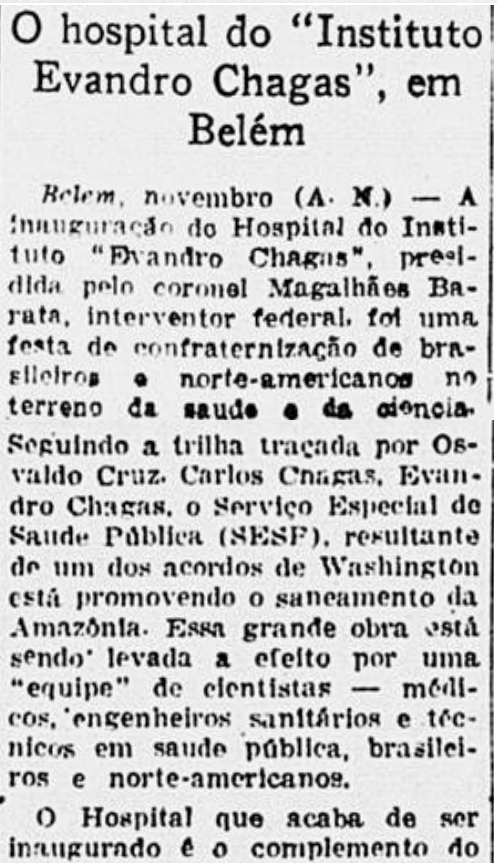 & 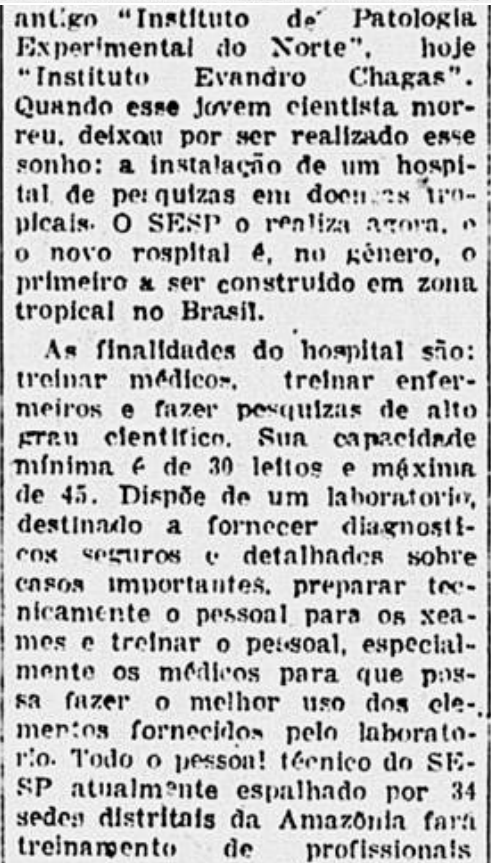 & 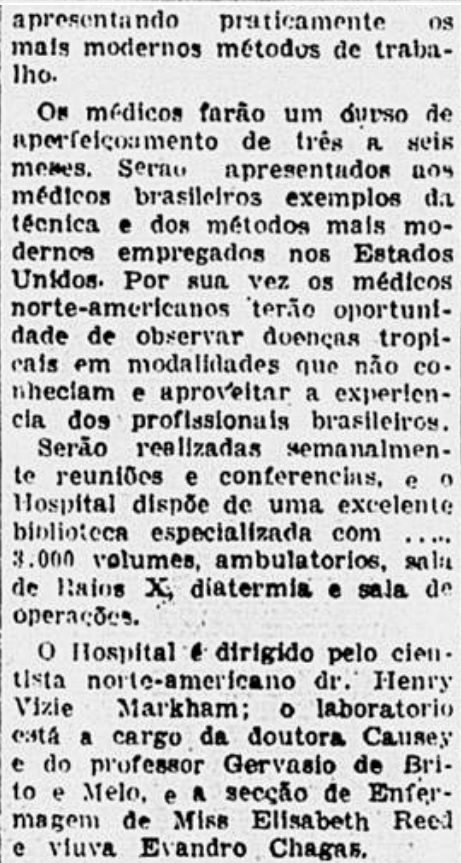 \\
\hline
\end{tabular}

Figura 3 - Adaptação da reportagem publicada no jornal carioca Correio da Manhã, em 1 de dezembro de 1943, sobre a inauguração do Hospital do Instituto Evandro Chagas

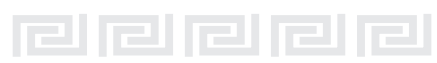

\section{REFERÊNCIAS}

1 Ivamoto HS. A Santa Casa de Misericórdia de Santos: sinopse histórica. Acta Med Misericordia. 1998 Jan: 1(1):7-10.

2 Landi AC, Sarmento P. Instituto Nacional de Infectologia Evandro Chagas. Rev Manguinhos. 2014 mai;29:38.

3 Chagas Filho C. Meu pai. Rio de Janeiro: Fiocruz; 1993. O pavilhão; p.233-46.

4 Pará. Lei n 59, de 10 de novembro de 1936. Crêa - Instituto de Pathologia Experimental do Norte e define suas attribuições. Belém: Officinas Graphicas do Instituto Lauro Sodré; 1936.

5 Bastos NCB. SESP/FSESP: 1942 - evolução histórica - 1991. Brasília: Fundação Nacional de Saúde; 1996. Hospital Evandro Chagas - Hospital de Belém; p. 412-7.
6 Deane LM. Histórico do Instituto Evandro Chagas: período de 1936-1949. In: Ministério da Saúde (BR). Fundação Serviços de Saúde Pública. Instituto Evandro Chagas: 50 anos de contribuição às ciências biológicas e à medicina tropical. Vol. 1. Belém: FSESP; 1986. p. 53-67.

7 saneamento da Amazônia. Observ Econom Finan. 1943 Set;8(92):45-55.

$8 \bigcirc$ hospital do "Instituto Evandro Chagas", em Belém. Correio da Manhã. 1943 dez 1;43(15056):2.

9 Campos ALV. Políticas internacionais de saúde na Era Vargas: o Serviço Especial de Saúde Pública, 1942-1960. Rio de Janeiro: Fiocruz; 2006. Organizando o trabalho: o exército de 'soldados da borracha'; p. 139-71.

10 Oliveira NNP. A borracha da Amazônia, os acordos de Washington e a política externa brasileira. In: Anais do $22^{\circ}$ Simpósio Nacional de História; 2003 jul 27-ago 1 ; João Pessoa, PB. São Paulo: Associação Nacional de História; 2003. p. 1-6. 